\title{
Holographic Cavalieri Principle as a Universal relation between Holographic Complexity and Holographic Entanglement Entropy
}

\author{
Davood Momeni ${ }^{1}$, Mir Faizal ${ }^{2,3}$, Ratbay Myrzakulov ${ }^{4}$ \\ ${ }^{1}$ Department of Physics, College of Science, Sultan Qaboos University, \\ P.O. Box 36, P.C. 123, Al-Khowd, Muscat, Sultanate of Oman \\ ${ }^{2}$ University of British Columbia - Okanagan \\ Kelowna, British Columbia V1V 1V7, Canada \\ ${ }^{3}$ Department of Physics and Astronomy, University of Lethbridge, \\ Lethbridge, Alberta, T1K 3M4, Canada \\ ${ }^{4}$ Eurasian International Center for Theoretical Physics \\ and Department of General Theoretical Physics, \\ Eurasian National University, Astana 010008, Kazakhstan
}

\begin{abstract}
In this paper, we will propose a universal relation between the holographic complexity (dual to a volume in AdS) and the holographic entanglement entropy (dual to an area in AdS). We will explicitly demonstrate that our conjuncture hold for all a metric asymptotic to $\mathrm{AdS}_{3}$, and then argue that such a relation should hold in general due to the AdS version of the Cavalieri principle. We will demonstrate that it holds for Janus solution, which have been recently been obtained in type IIB string theory. We will also show that this conjecture holds for a circular disk. This conjecture will be used to show that the proposal that the complexity equals action, and the proposal that the complexity equal volume can represent the same physics. Thus, using this conjecture, we will show that the black holes are fastest computers, using the proposal that complexity equals volume.
\end{abstract}

\section{Introduction}

Various studies done in different areas of physics have indicated that the laws of physics can be represented in terms of the ability of an observer to process relevant information 1, 2. Entropy measures the amount of information that is lost in a process, and hence, it is thought to be one of the most important quantities associated with any such information theoretical process. The entropy has been used to model physical phenomena from condensed matter physics to gravitational physics. Even the geometry of spacetime can be viewed as an emergent structure, which emerges due to an information theoretical process. This is because in the Jacobson formalism, the Einstein equation can be derived 
from thermodynamics by assuming a certain scaling behavior of the entropy 3, 4. This scaling behavior of entropy is that the maximum entropy of a region of space scales with its area, and this observation has been obtained using the physics of black holes. This observation has also led to the development of the holographic principle [5, 6, and the AdS/CFT correspondence is one of the most important realizations of the holographic principle [7.

The black hole information paradox occurs due to the observation that black holes are maximum entropy objects and they evaporate due to Hawking radiation. It is interesting to note that quantum entanglement has been used to discuss the microscopic nature of black hole entropy, with the hope that it may resolve the black hole information paradox [8, 9]. The AdS/CFT correspondence makes it possible to quantify quantum entanglement in terms of the holographic entanglement entropy [10, 11, 12. The entanglement entropy has been used in various branches of physics from quantum computing to condensed matter physics, and AdS/CFT correspondence make it possible to calculate it holographically. The holographic entanglement entropy of a CFT is dual to the area of a minimal surface defined in the bulk of an asymptotically AdS spacetime. So, for subsystem $A$ with its complement, it is possible to write an expression for the holographic entanglement entropy as

$$
S_{A}=\frac{\mathcal{A}\left(\gamma_{A}\right)}{4 G_{d+1}}
$$

where $G$ is the gravitational constant in the AdS spacetime, $\gamma_{A}$ is the $(d-1)$ minimal surface extended into the AdS bulk with the boundary $\partial A$, and $\mathcal{A}\left(\gamma_{A}\right)$ is the area of this minimal surface. It may be noted that usually there are UV divergence in holographic entanglement entropy, and so we need to use a regularization method to remove these divergences. Thus, for a deformed geometry, we define the area in this paper as,

$$
\mathcal{A}\left(\gamma_{A}\right)=\mathcal{A}_{D}\left(\gamma_{A}\right)-\mathcal{A}_{A d S}\left(\gamma_{A}\right)
$$

where $\mathcal{A}_{D}\left(\gamma_{A}\right)$ is the defined in deformed geometry (for example the geometry of a black hole), and $\mathcal{A}_{A d S}\left(\gamma_{A}\right)$ is defined in the background $A d S$ spacetime. Thus, we define the holographic entanglement entropy for a deformed geometry by subtracting the contribution coming from the background $A d S$ spacetime. This removes the divergent part and we are only left with a finite part. We will use this finite part in this paper, and call it the holographic entanglement entropy.

However, the recent studies have indicated that it is not enough to know what part of the information can be obtained by an observer from a system, but it is also important to know how difficult is it to obtain that information. As the entropy quantifies the abstract notion of the loss of information, the complexity quantifies the abstract notion of the difficulty to obtain the information (even if it is present in the system). The complexity (like entropy) has been used to study physical systems from black holes to condensed matter physics, and even quantum computing. In fact, recently it has been proposed that the information may not be ideally lost in a black hole, but it may be lost for all practice purposes as it would be impossible to reconstruct it from the Hawking radiation [13. As complexity has only been recently used to study various physical systems, there are different proposals to define the complexity for a CFT. However, recently 
motivated by holographic entanglement entropy, holographic complexity has been holographically defined as a quantity dual to a volume of codimension one time slice in anti-de Sitter (AdS) [14]-19. Furthermore, it is possible to use a subsystem $A$ with its complement, and define this volume as $V=V\left(\gamma_{A}\right)$, i.e., the volume enclosed by the same minimal surface which was used to calculate the holographic entanglement entropy [20,

$$
\mathcal{C}_{A}=\frac{V\left(\gamma_{A}\right)}{8 \pi R G_{d+1}}
$$

where $R$ and $V\left(\gamma_{A}\right)$ are the radius of the curvature and the volume in the AdS bulk. So, we will use this definition of the holographic complexity, and investigate a relation between this definition of holographic complexity and holographic entanglement entropy. It may be noted that just like the minimal area, this volume also contains UV divergences, and so we need to regularize this volume. So,for a deformed geometry, we define the volume as

$$
V\left(\gamma_{A}\right)=V_{D}\left(\gamma_{A}\right)-V_{A d S}\left(\gamma_{A}\right)
$$

where $V_{D}\left(\gamma_{A}\right)$ is the volume in deformed geometry, and $V_{A d S}\left(\gamma_{A}\right)$ is the volume in the background $A d S$ spacetime. So,we regularize the volume in a deformed geometry by subtracting the contribution coming from the background $A d S$ spacetime. This again removes the divergent part and we are again left with a finite part. In this paper, we will use this finite part of the holographic complexity.

Now as both the holographic complexity and holographic entanglement entropy are calculated using the same minimal surface, we expect that a universal relation to exist between them due to an AdS version of Cavalieri principle. In this letter, we will explicitly demonstrate this to be the case, and also find the explicit form of this universal relation. This can be used as a new holographic dictionary to calculate the holographic complexity from holographic entanglement entropy for different asymptotic AdS spacetimes. As both holographic entanglement entropy and complexity are used in various different branches of physics ranging from black hole physics to condensed matter physics, this general conjecture can have a lot of applications in those branches. This is because it is easier to calculate the entanglement entropy than complexity for various complex systems, and if this conjecture holds in general, this can be used as a holographic dictionary to obtain such quantities. Furthermore, as it is difficult to define complexity for the boundary theory, and a precise definition for complexity does not exist for the boundary theory, we will use this relation to obtain a definition for complexity of the boundary theory. This is because the complexity will be defined in terms of quantities whose boundary dual is well understood.

\section{Excited States in Bulk Geometry}

In this section, we will motivate a universal relation between the holographic complexity and holographic entanglement entropy. Now we consider an excited state in a $d+1$ dimensional conformal field theory (CFT), and assume it to be almost static and translational invariant. We want to analyse its gravity dual, 
so we write the metric on $A d S_{d+2}$ as 21

$$
d s^{2}=\frac{R^{2}}{z^{2}}\left[-f(z) d t^{2}+\frac{d z^{2}}{h(z)}+\sum_{i=1}^{d}\left(d x_{i}\right)^{2}\right] .
$$

Near the boundary $z \rightarrow 0$, we can assume $h(z) \simeq f(z) \simeq 1-\left(\frac{z}{z_{0}}\right)^{d+1}$, where $z_{0}$ is constant.

Let us consider an entangling region (subsystem $A$ ) in the shape of a strip defined by $0<x_{1}<l, \quad-L / 2<x_{2,3, \ldots, d}<L / 2$, where $L$ is taken to be infinite. So, we can parameterize the minimal surface $\gamma_{A}$ by $x_{1}=x(z)$, and write its area as

$$
\mathcal{A}\left(\gamma_{A}\right)=2 L^{d-1} R^{d} \int \frac{d z}{z^{d}} \sqrt{\frac{1}{h(z)}+x_{1}^{\prime}(z)^{2}} .
$$

where the derivative with respect to the $z$ is denotes by '. We can determine the shape of $x(z)$ by minimizing this area function. We note that the Lagrangian in Eq. (6) is independent of $x_{1}(z)$, and so the first integral associated with Euler-Lagrange equation can be expressed as

$$
x_{1}^{\prime}(z)=\frac{1}{h(z)} \frac{\left(\frac{z}{z^{*}}\right)^{d+1}}{\sqrt{1-\left(\frac{z}{z^{*}}\right)^{2(d+1)}}} .
$$

here we have assumed that $x_{1}^{\prime}\left(z^{*}\right)=\infty$. The total entangled length $l$, the entanglement area functional $\mathcal{A}\left(\gamma_{A}\right)$ and volume of codimension one time slice $V\left(\gamma_{A}\right)$ of the metric (5) are given by

$$
\begin{aligned}
& l=2 \int_{\epsilon}^{z^{*}} d z\left(\frac{z}{z^{*}}\right)^{d} \sqrt{\frac{1}{h(z)\left(1-\left(\frac{z}{z^{*}}\right)^{2 d}\right)}} \\
& \mathcal{A}\left(\gamma_{A}\right)=\frac{L^{d-1} R^{d}}{\left(z^{*}\right)^{d}} \int_{\frac{\epsilon}{z^{*}}}^{1} \frac{d \xi}{\xi^{d+1}} \sqrt{\frac{1}{h(\xi)\left(1-\xi^{2(d+1)}\right)}} \\
& V\left(\gamma_{A}\right)=\frac{L^{d-1} R^{d}}{\left(z^{*}\right)^{d-1}} \int_{\frac{\epsilon}{z^{*}}}^{1} \frac{x_{1}(\xi) d \xi}{\xi^{d+1} \sqrt{h(\xi)}} .
\end{aligned}
$$

It may be noted that to evaluate integral involving $l$, we have defined $\xi=\frac{z}{z^{*}}$, $h(z) \sim 1-\xi^{d+1} a^{d+1}$, and $a=\frac{z^{*}}{z_{0}}$. Now in general, $\epsilon \rightarrow 0$, thus it is adequate to just evaluate integral for dominant terms in the region $\xi \ll 1$. Using a series expansion in integrand, which is valid for all $\frac{\epsilon}{z^{*}}<\xi \ll 1$, we obtain,

$$
l \approx \frac{2 z^{*}}{d+1}\left(1-\left(\frac{\epsilon}{z^{*}}\right)^{d+1}\right)+\frac{z^{*} a^{d+1}}{2(d+1)}\left(1-\left(\frac{\epsilon}{z^{*}}\right)^{2(d+1)}\right) .
$$

In limit $\epsilon \rightarrow 0$, we obtain finite and regular length,

$$
l \approx \frac{z^{*}}{d+1}\left(1+\frac{a^{d+1}}{2}\right) .
$$

We can write the above equation in the following equivalent form,

$$
\begin{aligned}
& l \approx 2 z^{*} b\left(d, z^{*}, z_{0}\right), \\
& b\left(d, z^{*}, z_{0}\right)=\frac{1}{2(d+1)}\left(1+\frac{a^{d+1}}{2}\right) .
\end{aligned}
$$


Now we can use the same technique to calculate the area functional. So, we can change the variable from $z$ to $\xi$, use the series expansion (which is valid for all $\frac{\epsilon}{z^{*}}<\xi \ll 1$ ), and obtain,

$$
\mathcal{A}\left(\gamma_{A}\right) \approx \frac{L^{d-1} R^{d}}{\left(z^{*}\right)^{d}}\left(-\frac{1}{d}\left(1-\left(\frac{\epsilon}{z^{*}}\right)^{-d}\right)+\frac{a^{d+1}}{2}\left(1-\left(\frac{\epsilon}{z^{*}}\right)^{d+1}\right)\right) .
$$

Thus, we obtain both the finite and the divergent parts for this area as,

$$
\mathcal{A}\left(\gamma_{A}\right) \approx \frac{L^{d-1} R^{d}}{\left(z^{*}\right)^{d}}\left(-\frac{1}{d}+\frac{a^{d+1}}{2}\right)+\frac{L^{d-1} R^{d}}{\left(z^{*}\right)^{d}} \frac{1}{d}\left(\frac{\epsilon}{z^{*}}\right)^{-d} .
$$

The area used to calculate the holographic entanglement entropy is regularized by subtracting the the background AdS geometry from the deformed AdS geometry. Thus, we use this regularization to remove the divergent part, and obtain the final expression for regularized $\mathcal{A}\left(\gamma_{A}\right)$ as

$$
\begin{aligned}
& \Delta \mathcal{A}\left(\gamma_{A}\right) \approx \frac{L^{d-1} R^{d}}{\left(z^{*}\right)^{d}}\left(-\frac{1}{d}+\frac{a^{d+1}}{2}\right)=\frac{L^{d-1} R^{d}}{\left(z^{*}\right)^{d}} \alpha\left(d, z^{*}, z_{0}\right), \\
& \alpha\left(d, z^{*}, z_{0}\right)=\left(-\frac{1}{d}+\frac{a^{d+1}}{2}\right) .
\end{aligned}
$$

Note that from $\alpha\left(d, z^{*}, z_{0}\right)$, we can observe that $z^{*} \neq z_{0}$.

We can also calculate volume by using the use the same technique. So, we can again change the variable from $z$ to $\xi$, and use the series expansion, to obtain

$$
x_{1}(\xi) \approx z^{*}\left(\frac{\xi^{d+2}}{d+2}+\frac{a^{d+1} \xi^{2 d+3}}{2 d+3}\right)
$$

Thus, using the series expansions (after integration), we obtain

$$
V\left(\gamma_{A}\right) \approx \frac{L^{d-1} R^{d}}{2(d+2)\left(z^{*}\right)^{d-2}}\left(1-\left(\frac{\epsilon}{z^{*}}\right)^{2}\right) .
$$

The volume in the deformed AdS geometry is again regularized by subtracting the background AdS geometry from it. So, the regularized volume can be written as

$$
\begin{aligned}
& \Delta V\left(\gamma_{A}\right) \approx \frac{L^{d-1} R^{d}}{\left(z^{*}\right)^{d-1}} \nu\left(d, z^{*}\right), \\
& \nu\left(d, z^{*}\right)=\frac{z^{*}}{2(d+2)}
\end{aligned}
$$

So, using these results along with the expression for the holographic entanglement entropy and holographic complexity, we obtain

$$
\frac{C_{A}}{S_{A}}=\frac{n_{d} l}{R},
$$

where the regularized value of the constant $n_{d}$ is given by

$$
n_{d}=\frac{\nu\left(d, z^{*}\right)}{4 \pi \alpha\left(d, z^{*}, z_{0}\right) b\left(d, z^{*}\right)} .
$$


It may be noted that the effective temperature (entanglement temperature) $T_{\text {ent }}$ is proportional to the inverse of length of the region, as $T_{\text {ent }}=c l^{-1} 21$. So, we obtain the following universal relation between the holographic entanglement entropy and holographic complexity

$$
\frac{T_{e n t} \mathcal{C}_{A}}{S_{A}}=\frac{n_{d} c}{R}
$$

It may be noted that this relation is only valid for small subregions. This is because equation used in deriving this relation is only valid, when $z<<z_{0}[21$. So, all the calculations have been done for such a small region, in which thermal equilibrium is required. It is a region near the AdS boundary, and thus the proposed universality conjecture is valid for such a region. We would also like to point out that the metric function $h(z)$ is constructed from bulk, and we do consider any quantum correction from a backreaction on the metric background function.

This is a universal relation between the holographic complexity and holographic entanglement entropy. It exists due to an AdS version of the holographic cavalieri principle, and so we can call this universal relation as the Holographic Cavalieri Principle Conjuncture, and explicitly state it as following: Let us assume that two regions exist between two parallel AdS slice, and these two regions are codimension two slice of an asymptotically AdS space, such that they have equal areas, and the CFT duals to these two regions have equal entangled temperature, then they will have equal holographic complexity.

This conjecture can have a lot of applications as it can also be used to obtain a definition of complexity for the boundary theory. There is no agreed definition of the complexity for the boundary theory, however, using this conjecture, we can define the complexity of the boundary theory. Thus, we can obtain the complexity for the boundary theory using $\mathcal{C}_{A}=c \cdot n_{d} S_{A} / R T_{\text {ent }}$, because the boundary dual of all these quantities except complexity is well defined. So, this relation can be used as a definition for the complexity of the boundary theory. It is also expected that the holographic complexity would be directly proportional to the holographic entanglement entropy, as the difficulty of obtaining the information from a system will increase as the amount of information lost from a system will increase. Thus, this result is something we would expect on physical grounds for the boundary theory.

\section{Circular Disk}

In this section, we will demonstrate that this conjecture holds for a circular disk. The holographic entanglement entropy 11 and holographic complexity [20] for such a geometry has already been analyzed. We would like to point out that due to our definition of the area and volume of the minimal surface, we will only use the finite part of the holographic entanglement entropy and holographic complexity. This is because these quantities are regularized by subtracting the contributions coming from the background AdS. Now using the bulk of $\mathrm{AdS}_{d+2}$, it is possible to define a sphere of radius $l$. So, we parametrize such a metric as

$$
d s^{2}=\frac{R^{2}}{r^{2}}\left(-d t^{2}+d r^{2}+d \rho^{2}+\rho^{2} d \Omega_{d-1}\right)
$$


The entangling region is represented by $\{t=0, r \leq l\}$, where $l$ is the radius of a circular disk. The area and volume functionals for the parametrization $\rho=\rho(r)$, can be written as

$$
\begin{aligned}
& \mathcal{A}\left(\gamma_{A}\right)=\Omega_{d-1} R^{d} \int_{\epsilon}^{l} d r \frac{\rho(r)^{d-1}}{r^{d}} \sqrt{1+\left(\frac{d \rho(r)}{d r}\right)^{2}}, \\
& V\left(\gamma_{A}\right)=\frac{\Omega_{d-1} R^{d+1}}{d} \int_{\epsilon}^{l} d r \frac{\rho(r)^{d}}{r^{d+1}} .
\end{aligned}
$$

Now we can write the solution of equation of motion describing this system as $\rho(r)=\sqrt{l^{2}-r^{2}}$. Using this solution, we can obtain an expression for Eqs. (2425),

$$
\begin{aligned}
& \mathcal{A}\left(\gamma_{A}\right)=\Omega_{d-1} R^{d} \int_{\epsilon}^{l} d r \frac{\left(l^{2}-r^{2}\right)^{d / 2-1}}{r^{d}} \\
& V\left(\gamma_{A}\right)=\frac{\Omega_{d-1} R^{d+1}}{d} \int_{\epsilon}^{l} d r \frac{\left(l^{2}-r^{2}\right)^{d / 2}}{r^{d+1}}
\end{aligned}
$$

Now we expand the above integrals in series,and use the suitable regularization for them. So, we are only left with the finite part of the volume and area functionals,

$$
\begin{aligned}
& \mathcal{A}\left(\gamma_{A}\right)=\Omega_{d-1} R^{d} \sum_{n=0}^{\infty} \frac{(1-d / 2)_{n}}{n !(2 n-d+1)}, \\
& V\left(\gamma_{A}\right)=\frac{\Omega_{d-1} R^{d} l}{d} \sum_{n=0}^{\infty} \frac{(-d / 2)_{n}}{n !(2 n-d)} .
\end{aligned}
$$

Now using (13), we obtain

$$
\begin{aligned}
& S_{A}=\frac{\Omega_{d-1} R^{d}}{4 G} \sum_{n=0}^{\infty} \frac{(1-d / 2)_{n}}{n !(2 n-d+1)} \\
& \mathcal{C}_{A}=\frac{\Omega_{d-1} R^{d} l}{8 \pi R G d} \sum_{n=0}^{\infty} \frac{(-d / 2)_{n}}{n !(2 n-d)}
\end{aligned}
$$

So, we can write the ratio of such terms as

$$
\frac{\mathcal{C}_{A}}{S_{A}}=\frac{c_{d} l}{R} .
$$

Thus, we observe that even for this geometry, the holographic entanglement entropy is propotional to the holographic complexity. This is the expression we expected from our conjecture. Thus, this conjecture holds for the such a geometry.

\section{Asymptotically AdS Spacetime}

We can try to argue that such a conjecture is justified for a general asymptotically $\mathrm{AdS}_{d+1}$. The appropriate form of the metric written in Fefferman-Graham coordinates is given by

$$
d s_{d+1}^{2}=\frac{R^{2}}{r^{2}}\left(d r^{2}+g_{\mu \nu} d x^{\mu} d x^{\nu}\right)
$$


We choose an entangled strip parametrized by $\left\{t=0, x_{1}=x(r) \in[-l / 2, l / 2], x_{i} \in\right.$ $[0, L], i=2, . ., d-1$, and write the area functional for this metric as

$$
\mathcal{A}\left(\gamma_{A}\right)=R^{d-1} \int^{d-2} x d r \frac{\sqrt{g(r)\left(1+G(r) x^{\prime 2}\right)}}{r^{d-1}}
$$

where $g \equiv\left|g_{i j}\right|, G(r)=g_{11}-\frac{g_{1 i} g_{j 1}}{g_{i j}}$. A conserved charge can be constructed using this area functional (for general $x(r)$ )

$$
\left(\frac{R}{r}\right)^{d-1} \frac{\sqrt{g(r)} G(r) x^{\prime}}{\sqrt{g(r)\left(1+G(r) x^{\prime 2}\right)}}=\left(\frac{R}{r^{*}}\right)^{d-1} \sqrt{g\left(r^{*}\right) G\left(r^{*}\right)}
$$

Thus, we obtain the following result,

$$
x(r)=\int d r \frac{\left(\frac{R}{r^{*}}\right)^{d-1} \sqrt{g\left(r^{*}\right) G\left(r^{*}\right)}}{\sqrt{G(r)\left(g(r) G(r)\left(\frac{R}{r}\right)^{2(d-1)}-g\left(r^{*}\right) G\left(r^{*}\right)\left(\frac{R}{r^{*}}\right)^{2(d-1)}\right)}}
$$

Total entangled length $l$ and the total volume $V\left(\gamma_{A}\right)$, can be written as

$$
\begin{aligned}
l & =2 \int_{0}^{r^{*}} \frac{\left(\frac{R}{r^{*}}\right)^{d-1} \sqrt{g\left(r^{*}\right) G\left(r^{*}\right)}}{\sqrt{G(r)\left(g(r) G(r)\left(\frac{R}{r}\right)^{2(d-1)}-g\left(r^{*}\right) G\left(r^{*}\right)\left(\frac{R}{r^{*}}\right)^{2(d-1)}\right)}} \\
V\left(\gamma_{A}\right) & =\int d^{d-2} x \int_{0}^{r^{*}} x(r)\left(\frac{R}{r}\right)^{d} \sqrt{\left|g_{i j}\right|} \sqrt{1+\frac{g_{1 i} g_{j 1}}{g_{i j}}} d r
\end{aligned}
$$

As it is not possible to explicitly calculate the holographic entanglement entropy for a general metric, we will simplify our analysis to the general form of the metric on the $\mathrm{AdS}_{3}$. The metric of any asymptotically $\mathrm{AdS}_{3}$ can be represented by Eq. (33) when $g_{\mu \nu}=h_{\mu \nu} r^{d}$, where $h_{\mu \nu}$ is a uniform metric. Thus, for such a metric, we can easily integrate Eq. (35), and obtain

$$
x(r)=\frac{1}{\sqrt{H} \cosh \left(\frac{r}{r^{*}}\right)}
$$

where $H=h_{11}-\frac{h_{1 i} h_{j 1}}{h_{i j}}$. Now for $\mathrm{AdS}_{3}$, we can obtain the area as Area $=$ $2 R \sqrt{\left|h_{\mu \nu}\right|}$, and so the entanglement entropy can be written as

$$
S_{A}=\frac{R \sqrt{\left|h_{\mu \nu}\right|}}{2 G_{3}}
$$

Similarly, we can calculation the volume, and express it as $V\left(\gamma_{A}\right)=R^{2} \sqrt{\left|h_{\mu \nu}\right|} \mathcal{N}$ where $\mathcal{N}=\int_{\epsilon}^{1} \frac{d \xi}{\xi \cosh (\xi)} \approx-\frac{19}{96}-\ln (\epsilon)+\mathcal{O}\left(\epsilon^{6}\right) \gg 1$, and so the holographic complexity can be written as

$$
\mathcal{C}_{A}=\frac{R \sqrt{\left|h_{\mu \nu}\right|} \mathcal{N}}{8 \pi G_{3}}
$$

Furthermore, the total length is given by

$$
l=\frac{2}{\sqrt{H}} \mathcal{B}
$$


where $\mathcal{B}=\int_{0}^{1} \frac{d \xi}{\xi \sqrt{\xi^{2}-1}}=-\frac{\pi}{2}-i \ln \frac{\epsilon}{2}+\mathcal{O}\left(\epsilon^{6}\right) \gg 1$. Note that $\sqrt{H} \sim \frac{1}{\sqrt{\left|h_{i j}\right|}}$. If we combine three equations and using the definition of the temperature, we obtain

$$
\frac{T_{\text {ent }} \mathcal{C}_{A}}{S_{A}}=\frac{\mathcal{N}}{8 \pi \mathcal{B R}}
$$

Even though we have explicitly calculated this for all asymptotically $\mathrm{AdS}_{3}$, we can follow the same algorithm and calculate these quantities for any asymptotically AdS metric. However, such calculation, even though conceptually straightforward, can become computational complicated. So, in the next section, we will demonstrate this conjecture holds for an important asymptotically AdS.

\section{Janus solution}

Now we will explicitly test this conjuncture for Janus solution. First of all, we will consider a $\mathrm{AdS}_{3}$ Janus solution 37. The Janus solution interpolates between two AdS spaces [29. This bulk model for this solution is defined by the following action,

$$
S=-\frac{1}{16 \pi G_{N}} \int d x^{3} \sqrt{g}\left(\mathcal{R}-g^{a b} \partial_{a} \phi \partial_{b} \phi+\frac{2}{R^{2}}\right) .
$$

The Janus solution is a three dimensional (actually the simplest analytic) member of the generally $A d S_{d}$-sliced domain walls with the isometry group $S O(d-$ $1,2)[23,24,25,26,27,28$. The metric for such a solution can be written as

$$
d s^{2}=e^{2 A(r)} g_{i j}(x) d x^{i} d x^{j}+e^{2 h(r)} d r^{2},
$$

where $g_{i j}(x)$ is a metric on $A d S_{d}$ with scale $R_{d}$. It may be noted that such a domain wall has also been obtained as a solution in the type IIB supergravity 29. This solution has no $r$-dependent matter fields except a flowing dilaton $\phi(r)$. This solution is regular, if parameters are chosen such that the rate of variation of the dilaton is sufficiently slow. We use the radial coordinate $r$ for which $h(r)=0$, so we take $\phi^{\prime}=c \exp (-d A(r))$, and by this the scalar equation of is also satisfied. Now the wall profile equation can be written as

$$
A^{\prime 2}=\left(1 / L^{2}\right)\left[1-e^{-2 A}+b e^{-2 d A}\right]
$$

The constant $b$ is related to $c$ by $b=\frac{\kappa^{2}}{d(d-1)} c^{2} R^{2}$. We have set $R_{d}=L$ for simplicity. So, when $b=0$, the solution gives pure $\mathrm{AdS}_{d+1}$,

$$
d s^{2}=\cosh ^{2}(r / L) g_{i j}(x) d x^{i} d x^{j}+d r^{2} .
$$

However, for $b \neq 0$, it is not possible to obtain a simple solution for Eq. (46), (unless $d=2$ and this is the Janus metric). The metric for $A d S_{2}$ sliced domain walls in $A d S_{3}$ can be written as

$$
d s^{2}=e^{2 A(r)} d s_{A d S_{2}}^{2}+d r^{2} .
$$

The explicit solution of the equations of motion is the Janus solution, is given by the metric

$$
\begin{aligned}
& d s^{2}=R^{2}\left(d y^{2}+f(y) d s_{A d S 2}^{2}\right), \\
& f(y)=\frac{1}{2}\left(1+\sqrt{1-2 \gamma^{2}} \cosh (2 y)\right),
\end{aligned}
$$


and the dilaton is given by the function

$$
\phi(y)=\gamma \int_{-\infty}^{y} \frac{d y}{f(y)}+\phi_{1},
$$

where $\gamma\left(\leq \frac{1}{\sqrt{2}}\right)$ is the parameter of Janus deformation.

The metric of $\mathrm{AdS}_{2}$ slice is given by

$$
d s_{A d S 2}^{2}=\left(d z^{2}+d x^{2}\right) / z^{2}, \quad \phi_{1}=\phi(-\infty),
$$

and it is dual to the coupling constant of the exactly marginal deformation for the ground state $\left|\Omega_{1}\right\rangle$. The value $\phi_{2}=\phi(\infty)$ for the other ground state $\left|\Omega_{2}\right\rangle$ is obtained by performing the integral give in Eq. (51). So, we have

$$
\phi_{2}-\phi_{1}=\sqrt{2} \arctan \left[\frac{1-\sqrt{1-2 \gamma^{2}}}{\sqrt{2} \gamma}\right] \simeq \gamma,
$$

when $\gamma \ll 1$. Now if the bulk extension of the surface is parameterized by $x=x(r)$, then the corresponding area is given by

$$
\mathcal{A}\left(\gamma_{A}\right)=\int \frac{R^{2} f(0)}{z^{2}} \sqrt{1+x^{\prime}(z)^{2}} d z .
$$

The minimal surface $x(z)$ is the solution of the following equation,

$$
\frac{x^{\prime}(z)}{z^{2} \sqrt{1+x^{\prime}(z)^{2}}}=\frac{1}{\left(z^{*}\right)^{2}},
$$

with the auxiliary boundary condition, $\left.x^{\prime}(z)\right|_{z=z^{*}}=\infty$. Now the solution of this equation can be written as

$$
x(z)=z^{*}(E(\xi, i)-F(\xi, i))
$$

where $\xi=\frac{z}{z^{*}}$, and $E(z, k), F(z, k)$ are elliptic functions,

$$
\begin{aligned}
& E(z, k)=\int_{0}^{z} \frac{\sqrt{1-k^{2} r^{2}}}{\sqrt{1-r^{2}}} d r \\
& F(z, k)=\int_{0}^{z} \frac{d r}{\sqrt{1-k^{2} r^{2}} \sqrt{1-r^{2}}} .
\end{aligned}
$$

Thus, minimizing the area, we obtain

$$
\mathcal{A}\left(\gamma_{A}\right)=\frac{R^{2} f(0)}{z^{*}} \int_{\frac{\epsilon}{z^{*}}}^{1} \frac{d \xi}{\xi^{2} \sqrt{1-\xi^{4}}}
$$

where $z^{*}$ is turning point and $\epsilon$ is a UV cut off. Now for the solution Eq. (56), the total entangled length $l$, the finite part of the entanglement area functional $A(\gamma)$ and volume of codimension one time slice $V\left(\gamma_{A}\right)$ of the metric are obtained as follows,

$$
\begin{aligned}
& l=2 z^{*}(E(1, i)-F(1, i)) \\
& \mathcal{A}\left(\gamma_{A}\right)=\frac{R^{2} f(0)}{z^{*}}\left(-1+\sum_{n=1}^{\infty} \frac{(1 / 2)_{n}}{n !(4 n-1)}\right) \\
& V\left(\gamma_{A}\right)=R^{2}\left(\frac{\pi}{4}+K(i)-E(1, i)\right) .
\end{aligned}
$$


Here $K(k)$ is another elliptic functions,

$$
K(k)=\int_{0}^{1} \frac{d r}{\sqrt{1-k^{2} r^{2}} \sqrt{1-r^{2}}}
$$

So, using Eqs. (60) 62), it is possible to explicitly demonstrate that the holographic Cavalieri principle holds for this solution,

$$
\frac{T_{\text {ent }} \mathcal{C}_{A}}{S_{A}}=\frac{\mathcal{N}}{R}
$$

where $\mathcal{N}=\frac{c \cdot n_{1}}{\pi n_{2} f(0)}$ is a numeric factor, and $n_{1}, n_{2}$ are given by

$$
\begin{aligned}
& n_{1}=\frac{\frac{\pi}{4}+K(i)-E(1, i)}{2(E(1, i)-F(1, i))}, \\
& n_{2}=-1+\sum_{n=1}^{\infty} \frac{(1 / 2)_{n}}{n !(4 n-1)} .
\end{aligned}
$$

Thus, we have been able to explicitly demonstrate that holographic Cavalieri principle hold for Janus solution. This can be used to analyse the holographic complexity for the boundary theory dual to the Janus solution. It may be noted that Janus solution is dual to an interesting field theoretical system. This is because the field theoretical system dual to Janus solution is a boundary spacetime divided by a codimension one defect [29]. A different Yang-Mills coupling exists in each of the two halves of this boundary spacetime. In fact, the the string theoretical configurations for this solution have also been analysed 29. The conformal perturbation theory has been used to analyse the quantum level conformal symmetry of the Janus solution [30. The holographic entanglement entropy for Janus solution has been calculated, and it has been used for analyzing the behavior of boundary theory dual to the Janus solution 31. The holographic complexity can also be used to analyse the behavior of the boundary theory dual to the Janus solution. So, it would be interesting to use the results of this paper to analyse the behavior of the boundary theory dual to Janus solution.

\section{Application}

In this paper, we have used a proposal for holographic complexity, which states that the holographic complexity of a system is equal to the volume enclosed by a minimal surface. There is another recent proposal for the holographic complexity and this proposal states that the holographic complexity of a system is equal to the bulk action, calculated on a Wheeler-DeWitt patch [32, 33]

$$
\mathcal{C}=\frac{A}{\pi \hbar},
$$

where $A$ is the bulk action evaluated on the Wheeler-deWitt patch with a suitable temporal boundary, and $\mathcal{C}$ is this holographic complexity obtained using this new proposal. It is possible to calculate the action on a Wheeler-DeWitt 
patch for such geometries, using the null boundaries of the Wheeler-DeWitt patch.

It has been argued that black holes saturate the bound for rate of change of complexity, and so for black holes the rate of change of complexity is given by [32, 36]

$$
\frac{d \mathcal{C}}{d t}=2 M,
$$

where $M$ is the mass of the black hole. Thus, it was argued that the black holes are the fastest computes.

We will demonstrate that this result can also be obtained from the other proposals for holographic complexity (in which holographic complexity is related to the volume), using the conjuncture presented in this paper. Thus, we will argue that both these proposals for holographic complexity can represent the same physics. However, here we will use the holographic complexity for a subregion, and we so we need to first define the growth for it. It has been demonstrated it is possible to define the growth of such a volume [34, 35], and so we can analyze such a growth of holographic complexity for such a subregion, in this paper.

It is known that the holographic entanglement entropy scales as $L^{2}$ (because of the definition of the area used to calculate it). Furthermore, the entanglement temperature can be represented as $T_{\text {ent }} \sim L^{-1}$, where $L$ the size of entangled region. So, $T_{\text {ent }} \mathcal{C}_{A}$ represents the time variation of $\mathcal{C}_{A}$. Now using the conjecture proposed in this paper, we obtain

$$
\frac{d \mathcal{C}_{A}}{d t} \approx \frac{S_{A}}{R} \sim L \sim \text { size of horizon } \leq 2 M
$$

where $t \approx T_{\text {ent }}^{-1}$, and we have also assumed that the entangled region size remains in the limit of $L \leq$ size of horizon. Thus, we would obtain such a time from the entanglement temperature of the dual conformal field theory. So, in the limiting case for black holes with mass $M$, we obtain

$$
\frac{d \mathcal{C}_{A}}{d t}=2 M
$$

We again conclude that the black holes are fastest computers, however, we have now obtained this result using the proposal that the holographic complexity equals to the volume. Thus, we have demonstrated that the complexity defined in Eq. (3) along with Eq. (22), produces the same physics as as the complexity defined in Eq. (66). So, the proposal that holographic complexity is equal to the action, and the proposal that the holographic complexity is equal to the volume represent the same physics.

\section{Conclusion}

In this letter, we propose that a non-trivial but universal relation exists between the holographic quantum complexity and the holographic entanglement entropy. As this relates a quantity which is dual to a volume in AdS to a quantity which is dual to an area in AdS, it can be considered as a holographic version of Cavalieri principle. Furthermore, in analogy with the usual Cavalieri principle, the regions 
analysed were assumed to exist between two parallel AdS slice. We argued that such a conjuncture should hold in general, as it is based on the AdS version of the cavalieri principle. We also explicitly demonstrated this to be the case for $\mathrm{AdS}_{3}$. However, as it is not possible to obtain a general expression for the holographic entanglement entropy, we made a conjecture that such a universal relation should hold. This is because the higher dimensional case would be conceptually similar to this case, however, they would be computationally more complicated. We demonstrated that this conjecture holds for a circular disk. We also explicitly demonstrate that our conjuncture hold for Janus solution, which has recently been obtained in type IIB string theory.

We also analyzed the relation between the proposal that the holographic complexity equal to the action and the holographic complexity equal to the volume. It was already demonstrated that the black holes are the fastest computers using the proposal which stated that the holographic complexity is equal to the action evaluated at the Wheeler-DeWitt patch [32, 33]. In this paper, we demonstrated that the black holes are the fastest computers using the proposals which states that the holographic complexity is equal to the volume. This has done by using the universal relation between the holographic complexity and holographic entanglement entropy proposed in this paper. Thus, we demonstrated that both these proposals represent the same physics.

It is possible to apply this conjuncture to various different excited AdS solutions. So, it would be interesting to analyse the consequences of this conjuncture, and use it for analyse the behavior of holographic complexity and holographic entanglement entropy for various different excited AdS states. We also proposed that this universal relation can be used to obtain a definition of complexity for the boundary conformal field theory. It would be interesting to analyse the implications of this relation for the fidelity susceptibility [37], as fidelity susceptibility can be used to analyse the quantum phase transitions in field theories dual to different deformed AdS solutions [38, 39, 40. It might be possible to obtain a relation between the fidelity susceptibility and holographic complexity, and then use this relation along with the results obtained in this paper to analyse the quantum phase transitions in different systems. It will also be interesting to analyse the consequences of this conjuncture on the black hole information paradox, as both holographic complexity has become more important than holographic entanglement entropy as it has been argued that information might not be ideally lost, but it might be impossible to recover this information from Hawking radiation [13. So, it seems holographic complexity might be used for understanding the black hole information paradox, and the results of this paper can be used for analysing the behavior of holographic complexity.

It may be noted that there are other ways to define this volume in the bulk, so various different proposals have been used for holographic complexity. The complexity can also be calculated from the maximal volume in the AdS which ends on the time slice at the AdS boundary [37. It has been demonstrated that this proposal corresponds to the fidelity susceptibility of the boundary conformal field theory, and so it is important for analyzing the quantum phase transitions 38, 39, 40. However, in this paper, we have not use such proposals, and restrict our analysis to the volume enclosed by the minimal surface used for calculating the holographic entanglement entropy [20. It would be interesting to analyze a relation between the holographic complexity, entanglement entropy and fidelity susceptibility. It may be noted that such a relation exists for a D3-brane 
geometry 41, but it will be interesting to investigate if such a relation exists for other deformed geometries. It would also be interesting to investigate this for time dependent geometries, as holographic complexity 42 and holographic entanglement entropy [43] have been studied for such geometries.

\section{References}

[1] K. H. Knuth , AIP Conf. Proc. 1305, 3 (2011)

[2] P. Goyal, Information 3, 567 (2012)

[3] T. Jacobson, Phys. Rev. Lett. 75, 1260 (1995)

[4] R. G. Cai and S. P. Kim, JHEP 0502, 050 (2005)

[5] G. 't Hooft, arXiv:gr-qc/9310026

[6] L. Susskind, J. Math. Phys. 36, 6377 (1995)

[7] J. M. Maldacena, Adv. Theor. Math. Phys. 2, 231 (1998)

[8] A. Strominger and C. Vafa, Phys. Lett. B 379, 99 (1996)

[9] J. M. Maldacena, JHEP 0304, 021 (2003)

[10] S. Ryu and T. Takayanagi, Phys. Rev. Lett. 96, 181602 (2006)

[11] S. Ryu and T. Takayanagi, JHEP 0608, 045 (2006)

[12] V. E. Hubeny, M. Rangamani and T. Takayanagi, JHEP 0707, 062 (2007)

[13] S. W. Hawking, M. J. Perry and A. Strominger, Phys. Rev. Lett. 116, 231301 (2016)

[14] L. Susskind, Fortsch. Phys. 64, 24 (2016)

[15] L. Susskind, Fortsch. Phys. 64, 24 (2016)

[16] D. Stanford and L. Susskind, Phys. Rev. D 90, 12, 126007 (2014)

[17] D. Momeni, S. A. H. Mansoori and R. Myrzakulov, Phys. Lett. B 756, 354 (2016)

[18] O. Ben-Ami and D. Carmi, arXiv:1609.02514 [hep-th].

[19] J. Couch, W. Fischler and P. H. Nguyen, arXiv:1610.02038 [hep-th].

[20] M. Alishahiha, Phys. Rev. D 92, 126009 (2015)

[21] J. Bhattacharya, M. Nozaki, T. Takayanagi and T. Ugajin, Phys. Rev. Lett. 110, $091602(2013)$

[22] D. Bak, M. Gutperle and S. Hirano, JHEP 0305, 072 (2003)

[23] G. L. Cardoso, G. Dall'Agata and D. Lust, JHEP 0203, 044 (2002)

[24] G. Lopes Cardoso, G. Dall'Agata and D. Lust, JHEP 0107, 026 (2001) 
[25] A. H. Chamseddine and W. A. Sabra, Phys. Lett. B 517, 184 (2001)

[26] A. H. Chamseddine and W. A. Sabra, Nucl. Phys. B 630, 326 (2002)

[27] S. L. Cacciatori, D. Klemm and W. A. Sabra, JHEP 0303, 023 (2003)

[28] K. Behrndt and M. Cvetic, Phys. Rev. D 65, 126007 (2002)

[29] D. Bak, M. Gutperle and S. Hirano, JHEP 0305, 072 (2003)

[30] A. B. Clark, D. Z. Freedman, A. Karch and M. Schnabl, Phys. Rev. D71, $066003(2005)$

[31] M. Gutperle and J. D. Miller, Phys. Rev. D 93, 026006 (2016)

[32] A. R. Brown, D. A. Roberts, L. Susskind, B. Swingle and Y. Zhao, Phys. Rev. Lett. 116, 191301 (2016)

[33] A. R. Brown, D. A. Roberts, L. Susskind, B. Swingle, and Y. Zhao, Phys. Rev. D 93, 086006 (2016)

[34] M. Christodoulou and C. Rovelli, Phys. Rev. D 91, 064046 (2015)

[35] M. Christodoulou and T. D. Lorenzo, Phys. Rev. D 94, 104002 (2016) .

[36] S. Lloyd, Nature 406, 1047 (2000)

[37] M. Miyaji, T. Numasawa, N. Shiba,T. Takayanagi, and K. Watanabe, Phy. Rev. Lett 115, 261602 (2015)

[38] H. T. Quan, Z. Song, X. F. Liu, P. Zanardi, and C. P. Sun, Decay of loschmidt echo enhanced by quantum criticality, Phys. Rev. Lett. 96, $140604(2006)$

[39] P. Zanardi and N. Paunkoviic, Phys. Rev. E 74, 031123 (2006)

[40] P. Zanardi, P. Giorda, and M. Cozzini, Phys. Rev. Lett. 99, 100603 (2007)

[41] D. Momeni, M. Faizal, S. Bahamonde, A. Myrzakul and R. Myrzakulov, arXiv:1608.08819 [hep-th]

[42] D. Momeni, M. Faizal, S. Bahamonde and R. Myrzakulov, Phys. Lett. B $762,276(2016)$

[43] V. E. Hubeny, M. Rangamani and T. Takayanagi, JHEP 0707, 062 (2007) 